\title{
Now it looks more real \\ A Study of Metrics and Resolution for the Calibration of Dynamic Simulation
}

\author{
Aurélien Brès ${ }^{1}$, Frédéric Amblard ${ }^{2}$, Jessen Page $^{2}$, Stefan Hauer ${ }^{1}$, Anna Shadrina ${ }^{1}$ \\ ${ }^{1}$ AIT Austrian Institute of Technology, Center for Energy, Vienna, Austria \\ ${ }^{2}$ HES-SO Valais-Wallis, Sion, Switzerland
}

\begin{abstract}
The calibration of dynamic building simulation is an often essential but challenging process. There are approaches to formulate it mathematically as the minimization of a discrepancy function between simulation and measurements. In these approaches, the discrepancy is usually defined based on the mean square error (MSE) between vectors of simulated and measured quantities. The MSE metric reflects the area between the corresponding curves, without accounting for the similarity of curve shapes. Thus, it may overestimate the discrepancy of quantities subject to strong variations, especially in the case of on/off behavior with hysteresis and phase shifts. The aim of this contribution is to investigate whether the MSE still represents an effective metric in such cases, and to examine the usefulness of an alternative metric. This alternative metric is based on dynamic time warping (DTW), which allows similar features to be matched with some distortion on the time axis. The question is elaborated with the example of a waterto-water heat pump, which may cycle on and off at a subhourly frequency. Eight calibration experiments are carried out, differing in model adequacy, time resolution and discrepancy definition. Results show the inability of MSE to assess model quality when applied to high-frequency measurements. In comparison, it can be argued that the alternative metric based on DTW better reflects model quality.
\end{abstract}

\section{Introduction}

Calibration refers to the adaptation of uncertain input parameters for simulation results to better coincide with empirical data. In the field of building performance simulation (BPS), numerous sources of uncertainties make calibration an often essential but challenging process. The goodness-of-fit of calibration is typically assessed with metrics based on the mean square error (MSE) between vectors of simulated and measured quantities. Such metrics are also used as objective functions in optimisation-based calibration. This contribution looks at simulation calibration at subhourly time steps with quantities subject to quick variations and hysteresis. It investigates whether the MSE represents an effective metric to assess model quality, with the example of a water-towater heat pump. The heat pump may cycle on and off at a frequency higher than the hourly resolution typical for building simulation. Eight calibration experiments are performed, differing in model adequacy, time resolution and discrepancy definition.

After a review of related work in simulation calibration and similarity measures for time series, the second section introduces an alternative calibration metric based on dynamic time warping. The third section presents the calibration experiments carried out to investigate the indicated issues with high-resolution calibration and test the alternative calibration metric. Results and discussion of these experiments are found in the fourth and fifth sections.

\section{Related work}

\section{Optimization-based simulation calibration}

Dynamic simulation engines and the increasing availability of high-resolution measurements should make it possible to calibrate simulation at sub-hourly timesteps, and so to assess how well phenomena with a short time scale - frequent in heating, ventilation and air conditioning (HVAC) controls - are modelled. Calibration approaches reported in the literature have moved from monthly utility data to hourly and subhourly data, and it has been recognized that a higher time resolution increases calibration difficulty (Coakley et al., 2014).

Approaches to the calibration of building performance simulation can be broadly classified as manual or automated (Coakley et al., 2014). In manual calibration, iterative adjustments to simulation inputs are carried out by users based on their personal understanding of simulation and possibly with the support of graphical visualization. These iterations may incur high costs, and the process may lack reproducibility. Automated processes have been proposed to avoid these drawbacks. One approach to automate calibration is to formulate it mathematically as the minimization of a discrepancy function between simulation and measurements (Sun and Reddy, 2006; 
Yang et al., 2016). This approach is not without difficulties. When using state-of-the-art simulation tools such as EnergyPlus (Crawley et al., 2001), the objective function is usually neither differentiable nor even continuous, and it is computationally expensive to evaluate (Wetter and Wright, 2004). The underdetermination of the optimization problem is a more general issue, meaning that available measurements are usually not enough to adjust all uncertain parameters (Sun and Reddy, 2006). At an even more fundamental level, the optimisation-based calibration approach relies on the definition of an appropriate objective function to minimize.

\section{Building simulation calibration metrics}

The same metrics, described in this paragraph, are often used both for assessing calibration quality in general and for performing optimization-based calibration. For each time step $i$, where $1 \leq i \leq n$, let $s_{i}$ be the simulation result and $m_{i}$ the measured value. Metrics which have been widely used in building simulation calibration are (Coakley et al., 2014):

- The mean square error (MSE) $\frac{\sum_{i=1}^{n}\left(s_{i}-m_{i}\right)^{2}}{n}$ and its square root (RMSE).

- The coefficient of variation of the root mean square error CVRMSE $\frac{\sqrt{\frac{\sum_{i=1}^{n}\left(s_{i}-m_{i}\right)^{2}}{n}}}{\bar{m}}$.

- The mean bias error (MBE) $\frac{\sum_{i=1}^{n}\left(s_{i}-m_{i}\right)}{\sum_{i=1}^{n} m_{i}}$.

MSE and CVRMSE are based on a direct comparison of $m_{i}$ and $s_{i}$ for each time step $i$. On the other hand, MBE has a limited significance as it only accounts for the differences in aggregated values.

Despite the wide use of these metrics, there is no definite agreement on which metrics should be used, and how they should be evaluated. The most popular criteria for the validation of calibrated models are the calibration requirements of ASHRAE Guideline 14 (ASHRAE, 2002). However, they are only applicable to limited cases. For hourly calibration, the requirements are $|\mathrm{MBE}| \leq 10 \%$ and CVRMSE $\leq 30 \%$. The way discrepancy measures are normalized was recently challenged by Chakraborty and Elzarka (2018), who proposed to use RN_RMSE, where the divisor used for normalizing RMSE is the range of measured values instead of the mean of measured values. Actually, CVRMSE, RMSE and RN RMSE are only different ways of normalizing the MSE, and the same results should be obtained by minimizing any one of these four metrics. In recent work, Garrett and New (2016) attempted to determine the relevance of calibration metrics by correlating input and output metrics. The obtained correlations were shown to be weak for all the investigated metrics.
Similarity measures for time series and dynamic time warping

Simulation and monitoring results being instances of time series, the search for alternative calibration metrics may benefit from a review of similarity measures used for the analysis of time series in general, on which there exists extensive literature. The most commonly used similarity measures for time series include "lock-step" measures such as the mean square error, in which the values of two time series are compared pairwise for each time step, but also "elastic" measures such as dynamic time warping introduced below (Ding et al., 2008).

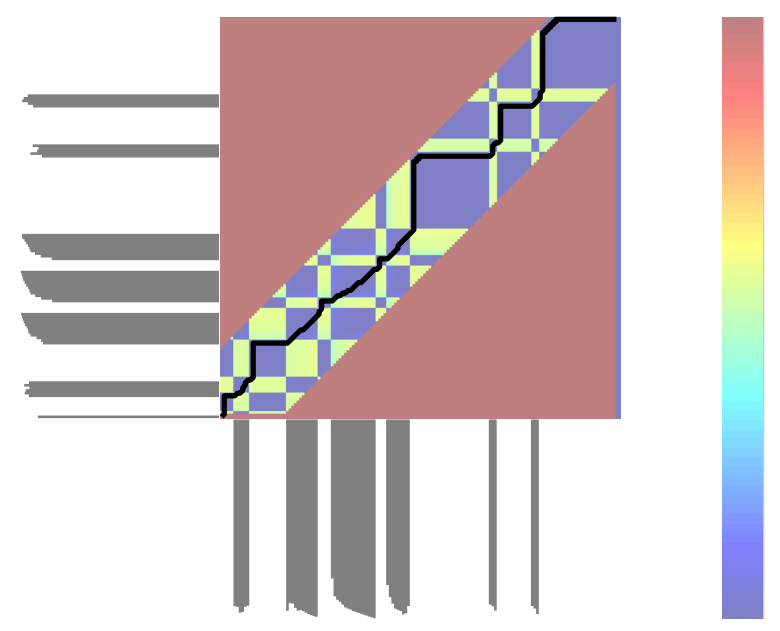

Figure 1: Minimum warping path (in black) for example time series $a$ and $b$ displayed in gray on the left and bottom, against values of $\left|a_{i}-b_{j}\right|^{2}$ displayed on the $n$-by-n square: low in blue, high in yellow, not allowed $(|i-j|>w)$ in red.

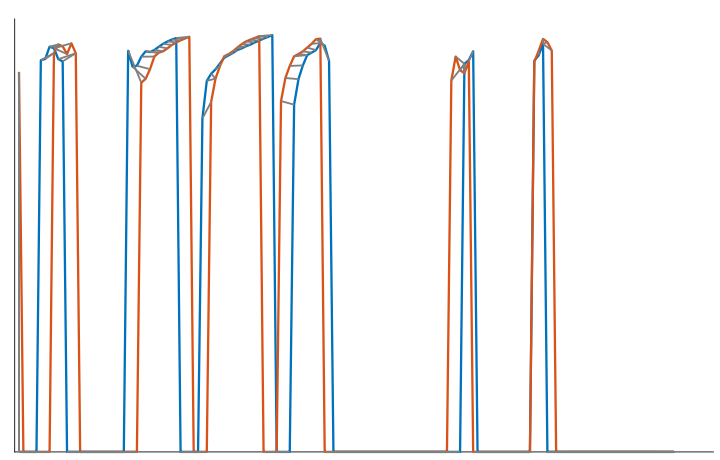

Figure 2: Dynamic time warping between the two time series of Figure 1, displayed as grey links between matched values.

Dynamic time warping (DTW) provides a measure of dissimilarity between two time series by calculating an optimal match between them (Lines and Bagnall, 2015). Initially used for spoken word recognition (Sakoe and Chiba, 1978), DTW is now used in 
a variety of applications, among others as a measure in state-of-the-art time series classification algorithms (Lines and Bagnall, 2015). Given two time series $a$ and $b$ of length $n$, one defines a warping path of length $n_{p} \geq n$ as a set of index pairs $\left(e_{k}, f_{k}\right)$ with $1 \leq k \leq$ $n_{p}$, such that $\left(e_{1}, f_{1}\right)=(1,1),\left(e_{n_{p}}, f_{n_{p}}\right)=(n, n)$ and $0 \leq e_{k+1}-e_{k} \leq 1$ and $0 \leq f_{k+1}-f_{k} \leq 1$ for all $1 \leq k \leq n_{p}$. The weight of such a path is defined as the sum of differences along this path: $\sum_{k=1}^{n_{p}}\left|a_{e_{k}}-b_{f_{k}}\right|^{2}$. The DTW distance is defined as the minimum weight of warping paths. One can view this as a minimum path between two diagonal ends of a matrix containing values of $\left|a_{i}-b_{j}\right|^{2}$, as illustrated in Figure 1. This minimum path can be found exactly in quadratic time, using dynamic programming (Salvador and Chan, 2007). Note that the values $a_{i}$ and $b_{i}$ of the time series may be scalar values, for instance the measured and simulated values $m_{i}$ and $s_{i}$ of one quantity, but also vector values, for instance combining several quantities.

One can restrict the allowed warping with a window length $w$, imposing $\left|e_{k}-f_{k}\right| \leq w$ for all $1 \leq k \leq$ $s$, which also reduces computation time. One may remark that the mean square error corresponds to the DTW distance with a window length of zero, and to a strictly diagonal path in the matrix view of Figure 1. In summary, DTW differs from the MSE in that it allows some shifting and distortion on the time axis.

\section{Approach}

\section{Problem statement}

The context of this work is the calibration of building performance simulation with detailed HVAC system modeling at sub-hourly time steps. In this context, control variables often take discrete (typically on/off) values, and are subject to hysteresis. As a result, simulated variables of interest exhibit non-smooth dynamics. The value of such a variable at a precise point in time may differ widely from the value at adjacent time steps, and is accordingly difficult to predict.

This issue is illustrated in Figure 3 with the comparison of simulation results obtained with two models differing only by a minor change in one input. Although the results can be said to be qualitatively similar, the discrepancy as measured by CVRMSE is very high $(161 \%)$. One reason for this is that slight shifts of peaks on the time axis are highly penalized by the mean square error, as errors of both signs (corresponding to unmixed red and blue in Figure 3) are added. Thus, the usefulness of calibration metrics based on a comparison of measured and simulated values time step by time step may be questioned.

\section{Alternative calibration metric based on dy- namic time warping}

In order to address the presented issues, an alternative calibration metric based on dynamic time warping is proposed. One motivation is the ability of

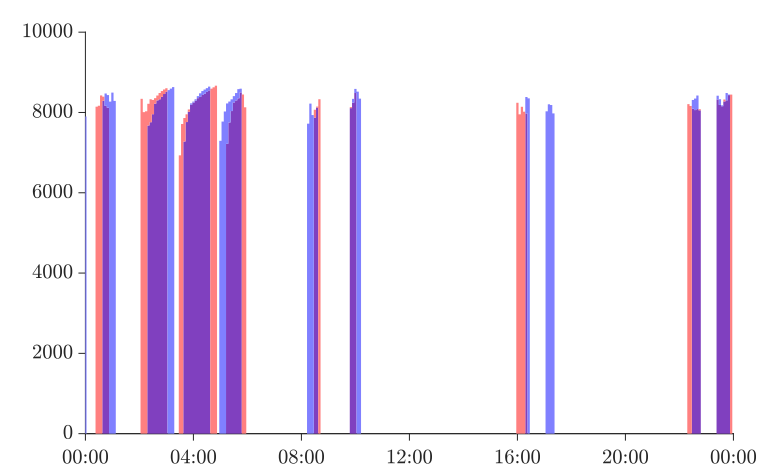

Figure 3: Results (one day with five-minute time steps) of two simulations differing by a slight variation (2\%) in design delivery mass flow rate. The comparison of these two simulations gives a CVRMSE value of $161 \%$. For comparison, the value of CVRMSE between the first vector of results and an all-zero vector would be $201 \%$.

DTW to "mitigate against distortions in the time axis" (Lines and Bagnall, 2015). The assumption is that DTW is a "better indicator" of the similarity of simulated and measured quantities than MSE. The quality of a measure of similarity is rather subjective and in general ill-defined. More particularly, we expect that DTW will be better than MSE at reflecting the quality of model aspects related to short-term system behavior. This model quality can be defined more objectively based on a knowledge of the existing system. This assumption will be tested by comparing calibration attempts with two different models, of which one can be said to be simpler to set up and the other to represent the real system control more faithfully.

Instead of simply taking the time series $m$ and $s$, let the time series considered for DTW be $m^{(k)}$ and $s^{(k)}$ which combine $k$ consecutive values such that, for $1 \leq$ $i \leq n-k, m_{i}^{(k)}$ is the vector $\left(m_{i}, m_{i+1}, \ldots, m_{i+k}\right)^{\top}$. This allows the matching to operate on more significant groups of values rather than on isolated values. Additionally, a maximum window length is used to make sure that values differing by more than a few hours cannot be matched.

Computing DTW is more expensive than computing MSE, but with detailed building and HVAC simulation, the limiting factor by far remains simulation time, so this does not represent an issue.

\section{Calibration experiments}

\section{Monitored system}

Experiments are carried out with the calibration of an existing heating system illustrated in Figure 5. The system serves a recently built multi-family building which is illustrated in Figure 4 and whose key parameters are summarized in Table 1 . Heat for space heating is provided by a water-water heat pump and stored in a buffer tank. The source side of the heat 
pump is connected to a local thermal network operated at a temperature level around that of ground water, also referred to as "anergy grid" (Buffa et al., 2019). Heat delivery to residential spaces uses floor heating with a design supply temperature of $35{ }^{\circ} \mathrm{C}$. Domestic hot water preparation uses a distinct heat pump and buffer tank and is not considered here. The performance of the system is monitored at 5-minutes intervals.

Table 1: Key parameters of the modelled building.

\begin{tabular}{|l|c|}
\hline Parameter & Value \\
\hline Location & Naters, Switzerland \\
Energy reference area & $2760 \mathrm{~m}^{2}$ \\
Construction year & 2011 \\
Norm energy demand for & $24 \mathrm{kWh} / \mathrm{m}^{2}$ \\
space heating & \\
$\begin{array}{l}\text { Nominal heat output of } \\
\text { space heating heat pump }\end{array}$ & $43.7 \mathrm{~kW}$ \\
\hline
\end{tabular}

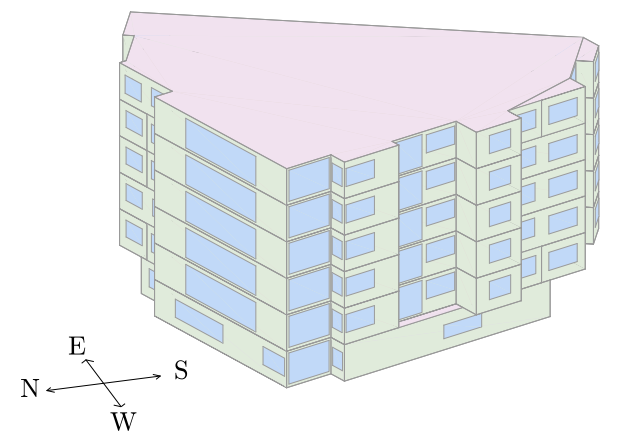

Figure 4: Building geometry.

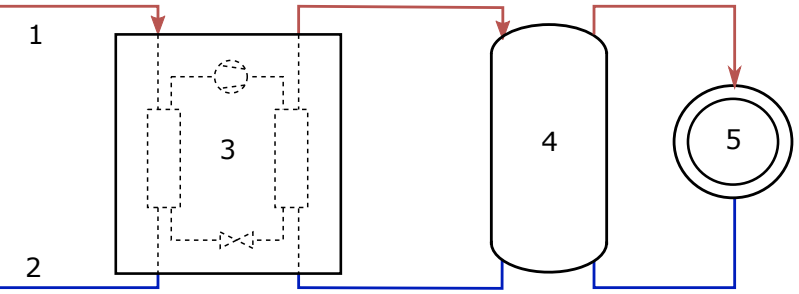

Figure 5: Heating system. 1: thermal network supply. 2: thermal network return. 3: heat pump circuit. 4: buffer tank. 5: floor heating.

The Waterkotte heat pump is remotely monitored using the Modbus protocol. Measurements of the heat pump are collected via a custom gateway and sent to an online platform for treatment and storage. The highest monitoring frequency in this case is up to one value per minute. However, values are updated only if a change is observed in order to reduce data transfer.

\section{Simulation model}

Dynamic simulation of the monitored system is carried out with EnergyPlus (Crawley et al., 2001). In order to minimize simulation time, and because the focus is on heating system modelling, the demand part is reduced to a one-zone model. The system model consists of three loops, which link the underfloor heating with the buffer tank, the buffer tank with the heat pump, and the heat pump with the water source, respectively.

The stratified tank control in the real system is based on two different measurement points: the heat pump starts when the temperature of the higher measurement point falls under a given cut-in temperature and shuts off when the temperature on the return side of the tank reaches a given cut-out temperature. In contrast, the default tank control model in EnergyPlus refers to a single tank node for cut-in and cut-out. Temperature control represents another discrepancy between the installed system and the default simulation setup. The real heat pump cycles on and off, with full capacity when it is on. In EnergyPlus, by default, heat pump power draw is modulated so that a given supply temperature is exactly reached.

In the following, results obtained with the default model (one-node control and modulating capacity) are compared to those obtained with a more realistic model (two-node control and full capacity). For the more realistic model, modifications are introduced with energy management system (EMS) advanced features of EnergyPlus (U.S. Department of Energy, 2016). This involves modifying the heat pump temperature setpoint at runtime such that full capacity is required.

Uncertain parameters are related to the building fabric, internal loads, heating system characteristics and heat pump control. A selection of uncertain parameters subjected to calibration is summarized in Table 2 .

Table 2: Uncertain parameters subjected to calibration and their ranges.

\begin{tabular}{|c|c|c|c|}
\hline Parameter & Min & Max & Unit \\
\hline $\begin{array}{l}\text { Difference between genera- } \\
\text { tion set point and storage } \\
\text { (cut-in) set point }\end{array}$ & 1.0 & 3.0 & $\mathrm{~K}$ \\
\hline $\begin{array}{l}\text { Difference between storage } \\
\text { cut-in and cut-out tempera- } \\
\text { ture }\end{array}$ & -1.5 & 1.5 & K \\
\hline Heating curve: y2 & 26 & 30 & ${ }^{\circ} \mathrm{C}$ \\
\hline $\begin{array}{l}\text { Occupant-defined heating } \\
\text { set point }\end{array}$ & 20 & 23 & ${ }^{\circ} \mathrm{C}$ \\
\hline $\begin{array}{l}\text { Heating delivery control } \\
\text { bandwidth }\end{array}$ & 0.8 & 1.4 & K \\
\hline Infiltration rate & 0.08 & 0.16 & $\mathrm{~h}^{-1}$ \\
\hline Storage cut-in node height & 0.6 & 0.9 & \\
\hline $\begin{array}{l}\text { Heat pump: ratio between } \\
\text { flow rates on source and de- } \\
\text { mand side }\end{array}$ & 1.2 & 1.6 & \\
\hline $\begin{array}{l}\text { Storage tank height to diam- } \\
\text { eter ratio }\end{array}$ & 1.5 & 2.5 & \\
\hline
\end{tabular}




\section{Optimization-based calibration}

Optimization-based calibration is carried out with particle swarm optimization (PSO), a gradient-free meta-heuristic method which is commonly used in BPS-based optimization (Nguyen et al., 2014; Yang et al., 2016).

The same optimization parameters are used for each calibration experiment, with 20 iterations and 20 particles. This optimization is implemented in the MATLAB environment, whereby the code also takes care of creating EnergyPlus simulation models corresponding to each set of input parameters and of computing the corresponding values of the objective function based on simulation results. The objective function subject to minimization is a measure of discrepancy between simulation and measurement results. This measure of discrepancy is, alternatively, CVRMSE, or DTW with a window size of four hours (or 48 5-minute time steps). Minimizing CVRMSE is assumed to give the same results as minimizing MSE or other metrics differing by their normalization. These measures of discrepancy are applied to heat pump power consumption in $\mathrm{W}$.

\section{Experiment design}

A key point of our problem statement is to compare mean square error (MSE) and dynamic time warping as calibration metrics. The time resolution at which these metrics are applied is assumed to play an important role. Also, model complexity is expected to have an effect on the discrepancy between simulation and reality.

Table 3: Calibration experiments with calibration time step duration in minutes, model and, where $D T W$ is applied as a calibration metric, parameters $w$ (window length) and $k$ (number of consecutive values compared).

\begin{tabular}{|l|r|r|l|l|}
\hline Experiment & Time step & w & k & Model \\
\hline mse-5m-ems & 5 & - & - & ems \\
mse-5m-def & 5 & - & - & def \\
mse-hr-ems & 60 & - & - & ems \\
mse-hr-def & 60 & - & - & def \\
dtw-5m-ems & 5 & 48 & 6 & ems \\
dtw-5m-def & 5 & 48 & 6 & def \\
dtw-hr-ems & 60 & 4 & 2 & ems \\
dtw-hr-def & 60 & 4 & 2 & def \\
\hline
\end{tabular}

$2^{3}=8$ calibration experiments are carried out in order to investigate the relations between these three factors, as summarized in Table 3, by modifying:

- The discrepancy measure, either mean square error (mse) or dynamic time warping (dtw).

- The calibration time resolution, whereby measurements and simulations are compared either for hourly (hr) or five-minute $(5 \mathrm{~m})$ time steps.

- The model structure, whereby heat pump control modelling is either as implemented in the
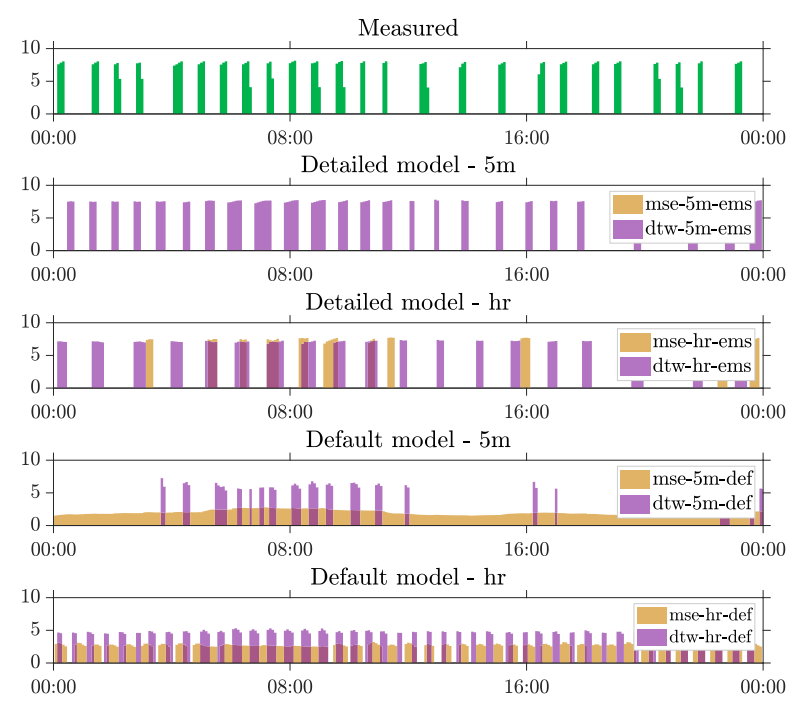

Figure 6: One-day view of calibrated results for compressor power in $k W$ with the eight calibration experiments, based on metrics CVRMSE (orange) and DTW (purple).

simulation tool by default (def) or more detailed and presumably more realistic, as made possible by the energy management system advanced features (ems).

Each calibration experiment corresponds to an optimization attempt, i.e. a few hundred simulation runs.

\section{Results}

The high number of time steps and high-frequency variations make the comparison of simulation results and measurements at subhourly time steps challenging. Such time series can be visualized better for shorter periods of time, for instance for one day as in Figure 6. It can be seen from these plots that, with the default model, the heat pump modulates its power to follow the heat demand and rarely shuts off. This is very different from the real system as measured, in which the heat pump cycles on and off with peaks lasting often less than one hour. The results of the detailed model shows an on/off behavior similar to the measurements, although the spacing of peaks is somewhat different. Strikingly, Figure 6 shows no heating for mse-5m-ems on the selected day. As confirmed by Figure $7 \mathrm{a}$ and Table 4, the MSE-optimized result for this case exhibits a strong negative bias, which can be interpreted in the following way: the penalization for misplaced peaks in this 5-minute calibration problem is so high that this solution with the heat pump off most of the time yields lower MSE values than other solutions.

Alternative visualizations such as the load duration curves in Figure 7 also allow the results to be condensed in a more intelligible form. These load duration curves confirm the idea that results obtained with the detailed model (Figure 7a) are "closer" to 
Table 4: Results for several calibration experiments (columns) in terms of different metrics (rows). Indicated are the results obtained with the detailed (Det) and the default model (Def), respectively.

\begin{tabular}{|c|cc|cc|cc|cc|}
\hline & \multicolumn{7}{|c|}{ Calibration experiments } \\
\hline & \multicolumn{2}{|c|}{ mse-5m } & \multicolumn{2}{c|}{ mse-hr } & \multicolumn{2}{c|}{ dtw-5m } & \multicolumn{2}{c|}{ dtw-hr } \\
& ems & def & ems & def & ems & def & ems & def \\
\hline CVRMSE 5m in \% & $\mathbf{1 8 4}$ & $\mathbf{1 5 7}$ & 208 & 163 & 227 & 196 & 215 & 187 \\
CVRMSE hr in \% & 107 & 47 & $\mathbf{8 0 . 9}$ & $\mathbf{4 3 . 3}$ & 81.1 & 84.9 & 69.8 & 49.2 \\
DTW 5m in \% & 40.2 & 38.9 & 27 & 37.4 & $\mathbf{2 0 . 3}$ & $\mathbf{2 9 . 5}$ & 24.4 & 34.1 \\
DTW hr in \% & 51.9 & 18.9 & 31.3 & 16.9 & 30 & 33.1 & $\mathbf{2 4 . 6}$ & $\mathbf{1 8 . 2}$ \\
CVRMSE daily in \% & 99.4 & 31.8 & 49.9 & 26.1 & 56.1 & 51 & 43.7 & 32.8 \\
MBE in \% & -98.2 & 1.99 & -15.6 & 2.23 & 31.9 & -20.5 & 15.3 & 8.06 \\
\hline
\end{tabular}

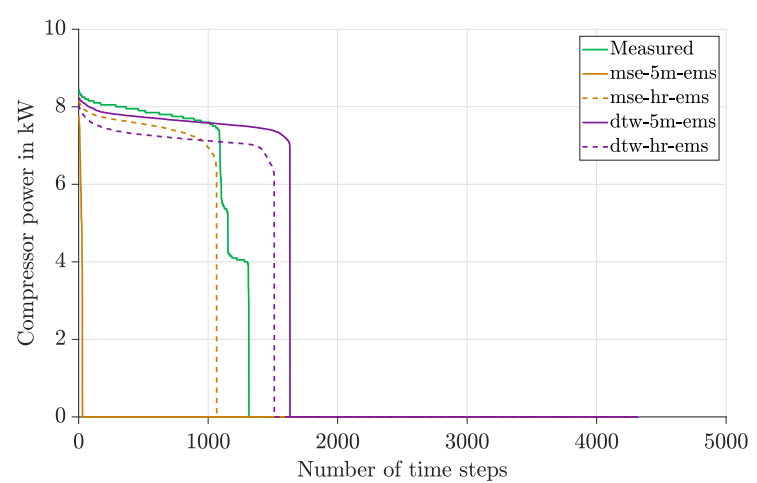

(a) Detailed model.

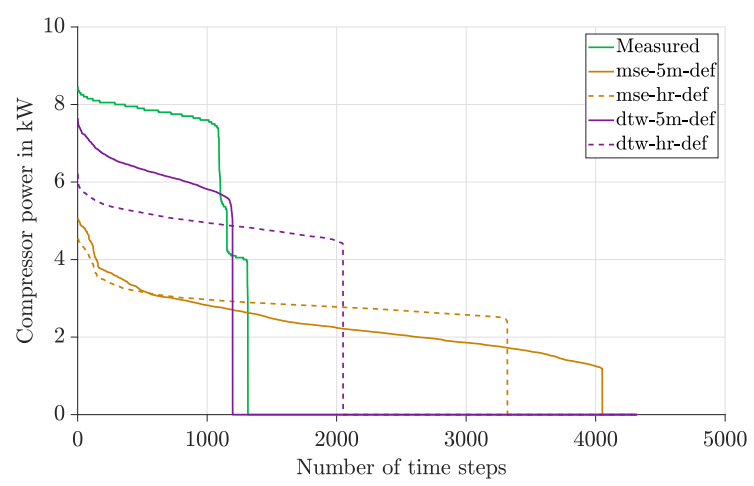

(b) Default model.

Figure 7: Load duration curves of calibrated results for the eight calibration experiments, with the detailed model (7a) and default model (7b), based on metrics CVRMSE (orange) and DTW (purple).

the measurements than those obtained with the default model (Figure 7b), except for the pathological case mse-5m-ems. When calibrating at fiveminute time steps (full lines), DTW-based calibration yields load duration curves closer to the measurements than MSE-based calibration. When calibrating with hourly values (dotted lines), results obtained with both metrics are more similar.

The results of the eight calibration experiments are summarized in Table 4. The values displayed in bold are of particular interest, as they correspond to the values subject to minimization in each experiment. Interestingly, the CVRMSE values obtained with the default model are lower than the ones obtained with the detailed model, especially at the five-minute resolution. This can be explained by the fact that the default model yields "smoother" curves, for which time shifts against the reference curve tend to be less penalized than in the case of curves with sharp peaks. As for optimized DTW values, they are significantly lower for the detailed model than for the default model at the five-minute resolution. This does not hold true for calibration at the hourly resolution.

Looking at the different metrics and plots above, the results obtained by minimizing MSE and DTW metrics can be seen to be more similar when calibration is done at the hourly resolution, and to differ more strongly at the five-minute resolution.

\section{Discussion}

Given the time scales of the monitored system behavior, it can be argued that considering sub-hourly results rather than hourly results makes a significant difference, and that it is instrumental in ensuring that the system is modelled correctly.

The CVRMSE values at the five-minute resolution are all above $150 \%$. Many of these values are not much better, and sometimes worse, than the one obtained with an all-zero vector (184\%). Accordingly, minimizing CVRMSE in some cases yields models with a large negative bias, as in the extreme case of mse-5m-ems. The CVRMSE values at the hourly resolution are all above $40 \%$, meaning that the model does not meet the corresponding requirement of ASHRAE guideline 14. One may argue that high CVRMSE values are simply a sign that the simulation model is not good enough.

One may point to limitations of the simulation model, even in its detailed version. These include the limited level of detail of the building model, the modeling of floor heating control, as well as the steady-state heat pump model. While the latter can give good results at hourly time steps, startup and shutdown effects become significant for shorter time intervals (Fuentes et al., 2016; Uhlmann and Bertsch, 2012).

However, the example presented in Figure 3 shows that very small parameter variations even from a perfect model would also yield high CVRMSE values for this calibration variable subject to strong vari- 
ations and hysteresis. Thus, it can be argued that in such cases CVRMSE applied to sub-hourly results is bound to give poor results, unless boundary conditions are very accurately monitored and modelled. For such calibration cases, dynamic time warping represents an interesting alternative. Contrary to CVRMSE, minimized DTW values are lower for the detailed model than for the default model, where the results of this detailed model can be seen to be qualitatively closer to the measurements (Figure 6). Also, calibration based on the alternative DTW metric does not yield aberrant results as obtained with MSE in the case of mse-5m-ems. These results support the assumption that the alternative calibration metric is a better choice than MSE in the case of sub-hourly results.

For hourly calibration, DTW and MSE metrics give more similar results, so that the use of DTW does not seem to be useful. However, this conclusion is dependent on the time scale of the observed system behaviour. For a slower system, for instance with a larger tank, DTW may also be useful for hourly calibration.

A drawback of the proposed DTW-based metric is that it uses parameters, in our case the window length $w$ and the number $k$ of consecutive values considered, for which appropriate values have to be chosen with regard to the specific problem. In fact, given the complexity of defining a meaningful and robust calibration metric, multi-objective calibration approaches, which have become popular in other modeling disciplines (Efstratiadis and Koutsoyiannis, 2010), could also make sense for building performance simulation in cases such as the one presented in this contribution.

\section{Conclusion}

This paper challenges pairwise comparison of simulated and measured values as the preferred way to define calibration metrics. An alternative measure of discrepancy has been provided, based on dynamic time warping. For calibration of heat pump power consumption at a time resolution of five minutes, this alternative metric gives preference to results obtained with a more detailed control model. Visual inspection of these simulation results also shows them to be qualitatively closer to the measurements. The CVRMSE metric, on the other hand, gives preference to results obtained with a default model, which are generally smoother but less representative of the measured behavior. Thus, it can be argued that the proposed metric based on dynamic time warping is more useful than the mean square error in assessing model quality, at least in the presented case.

Several directions for further work on the topic of calibration metrics can be inferred from the present study. Firstly, calibration using several data points simultaneously would be an interesting application of dynamic time warping. Secondly, calibration experiments with synthetic data, in which a ground truth for input parameters is available, may allow the value of different metrics to be investigated more objectively. Finally, the present contribution suggests that building performance simulation may in certain cases benefit from a multi-objective calibration approach.

\section{Acknowledgment}

This research was performed within the SIM4BLOCKS project (funded from the European Union's Horizon 2020 research innovation program under grant agreement No. 695965).

\section{References}

ASHRAE (2002). ASHRAE Guideline 14 - Measurement of Energy and Demand Savings.

Buffa, S., M. Cozzini, M. D'Antoni, M. Baratieri, and R. Fedrizzi (2019). 5th generation district heating and cooling systems: A review of existing cases in europe. Renewable and Sustainable Energy Reviews $104,504-522$.

Chakraborty, D. and H. Elzarka (2018). Performance testing of energy models: are we using the right statistical metrics? Journal of Building Performance Simulation 11(4), 433-448.

Coakley, D., P. Raftery, and M. Keane (2014). A review of methods to match building energy simulation models to measured data. Renewable and sustainable energy reviews 37, 123-141.

Crawley, D. B., L. K. Lawrie, F. C. Winkelmann, W. F. Buhl, Y. J. Huang, C. O. Pedersen, R. K. Strand, R. J. Liesen, D. E. Fisher, M. J. Witte, and Others (2001). EnergyPlus: creating a newgeneration building energy simulation program. Energy and buildings 33(4), 319-331.

Ding, H., G. Trajcevski, P. Scheuermann, X. Wang, and E. Keogh (2008). Querying and mining of time series data: experimental comparison of representations and distance measures. Proceedings of the VLDB Endowment 1(2), 1542-1552.

Efstratiadis, A. and D. Koutsoyiannis (2010). One decade of multi-objective calibration approaches in hydrological modelling: a review. Hydrological Sciences Journal-Journal Des Sciences Hydrologiques 55(1), 58-78.

Fuentes, E., D. Waddicor, and J. Salom (2016). Improvements in the characterization of the efficiency degradation of water-to-water heat pumps under cyclic conditions. Applied energy 179, 778-789.

Garrett, A. and J. R. New (2016). Suitability of ashrae guideline 14 metrics for calibration. ASHRAE Transactions 122, 469-477. 
Lines, J. and A. Bagnall (2015). Time series classification with ensembles of elastic distance measures. Data Mining and Knowledge Discovery 29(3), 565592.

Nguyen, A.-T., S. Reiter, and P. Rigo (2014). A review on simulation-based optimization methods applied to building performance analysis. Applied Energy 113, 1043-1058.

Sakoe, H. and S. Chiba (1978). Dynamic programming algorithm optimization for spoken word recognition. IEEE transactions on acoustics, speech, and signal processing 26(1), 43-49.

Salvador, S. and P. Chan (2007). Toward accurate dynamic time warping in linear time and space. Intelligent Data Analysis 11(5), 561-580.

Sun, J. and T. A. Reddy (2006). Calibration of building energy simulation programs using the analytic optimization approach (rp-1051). HVACER Research 12(1), 177-196.

Uhlmann, M. and S. S. Bertsch (2012). Theoretical and experimental investigation of startup and shutdown behavior of residential heat pumps. International Journal of Refrigeration 35(8), 2138 2149 .

U.S. Department of Energy (2016). EnergyPlus Documentation - Application Guide for EMS.

Wetter, M. and J. Wright (2004). A comparison of deterministic and probabilistic optimization algorithms for nonsmooth simulation-based optimization. Building and Environment 39(8), 989-999.

Yang, T., Y. Pan, J. Mao, Y. Wang, and Z. Huang (2016). An automated optimization method for calibrating building energy simulation models with measured data: Orientation and a case study. Applied energy 179, 1220-1231. 